\title{
Spatial properties of the complex decameter type II burst observed
} on 31 May 2013

\author{
Vladimir Dorovskyy ${ }^{1}$, Valentin Melnik ${ }^{1}$, Alexander Konovalenko ${ }^{1}$, \\ Anatoly Brazhenko ${ }^{2}$, Helmut Rucker ${ }^{3}$ \\ ${ }^{1}$ Institute of Radio Astronomy of NASU, Kharkov, Ukraine, \\ 2 Poltava Gravimetric Observatory of NASU, Poltava, Ukraine, \\ ${ }^{3}$ Commission for Astronomy of AAS, Graz, Austria.
}

E-mail: vlad.dorovskyy@gmail.com

Accepted: 18 December 2017

\begin{abstract}
We present the results of observations of a complex and powerful type II radio burst associated with a narrow Earth-directed coronal mass ejection (CME), which was ejected at around 11 UT on 31 May 2013. The observations were performed by the radio telescope UTR-2, which operated as a local interferometer, providing the possibility of detection of the spatial parameters of the radio emission source. There are also polarization data from URAN-2 radio telescope. The CME was detected by two space-born coronagraphs SOHO/LASCO/C2 and STEREO-BEHIND/COR1, and was absolutely invisible to the STEREO-AHEAD spacecraft.

The associated type II burst consisted of two successive parts of a quite different appearance on the dynamic spectrum. The first burst was narrow in frequency, had a cloudy structure and was completely unpolarized, while the second one showed a rich herring-bone structure and exposed a high degree of circular polarization. Both parts of the type il burst reveal band splitting and a well-distinguished harmonic structure.

The positions and sizes of the sources of the type II burst were found using cross-correlation functions of interferometer bases.

The sources of the type II burst elements were found to be of about 15 arcmin in size on average, with the smallest ones reaching as low as $10 \mathrm{arcmin}$. Corresponding brightness temperatures were estimated. In most cases these temperatures were between 1011 and $1012 \mathrm{~K}$ with maximum value as high as $1014 \mathrm{~K}$.

The angular displacement of the source of the type II back-bone was found to correspond to the linear velocity of about $9 \cdot 107 \mathrm{~cm} / \mathrm{s}$. At the same time the sources of the herring-bone sub-bursts moved much faster, about $1010 \mathrm{~cm} / \mathrm{s}$. Moreover the sources of positively drifting sub-bursts moved towards the Sun and the sources of negatively drifting counterparts moved away from the Sun. The heights and the velocities of the sources obtained from the interferometric observations were in quite good agreement with those obtained from the frequency drift rates assuming the Newkirk corona density model.
\end{abstract}

(c) 2018 BBSCS RN SWS. All rights reserved

Keywords: Solar radio emission, type II burst, herringbone structure, decameter wavelengths, radio source size.

\section{Introduction}

Solar type II radio bursts are manifestations of the coronal mass ejection (CME) movement through the solar corona and interplanetary space, and they originate from the shock formed ahead of a CME. The CMEs, in turn, are the most effective drivers of the space weather changes. Though type II bursts are observed in a wide frequency range, the bursts detected at meter and decameter-hectometer wavelengths are believed to be connected with the potentially most geoeffective CMEs (Gopalswamy et al. 2005).

The geoeffectiveness of the CME is mostly defined by its mass, velocity and direction. The velocity of the CME can be detected in three ways: by coronagraph observations in visible light, by the frequency drift rate of type II bursts and by the speed of the type II source displacement. The first method gives correct results for CMEs that propagate close to the sky plane and it is not applicable for Earth-directed CMEs (when observing from Earth). This problem can be solved by multi-spacecraft observations such as those provided by the STEREO mission. In addition, multi-spacecraft observations also allow to define the direction of the CME movement. However, the accuracy of this method depends on mutual positions of the STEREO satellites with respect to Earth and these positions are changing with time. The second method is indirect and model dependent one. In addition, it gives the radial component of the velocity vector and is inaccurate for CMEs that move at a substantial angle to the density gradient. This method is especially effective in cases of Earth-directed CMEs. The third method deals with the spatial displacement of the source of type II burst with time on the sky plane regardless of the chosen coronal density model, though it suffers from the projection effects.

It is also known that type II bursts reveal a wide variety of morphological features, such as fundamental and harmonic emission, band splitting, a cloudy structure, a herring-bone (HB) structure etc. (Nelson and Melrose, 1985; Melnik et al. 2004; Dorovskyy et al. 2015). The interpretations of all these features are based on their spectral properties assuming some models of plasma distributions in the corona and the shock. For instance, the band splitting is attributed to the plasma emission from regions ahead (upstream) and behind (downstream) the shock (Vršnak et al. 2004), HB sub-bursts are manifestations of shock-accelerated electrons escaping from the shock towards and away from the Sun (Cairns and Robinson, 1987). Spatial measurements with a high time, frequency and spatial resolution may confirm or call these assumptions into question.

Thus, our study of the spatial properties of the sources of type II bursts may improve the quality and reliability of the space weather diagnostics and will 
lead to better understanding of underlying physics as well.

\section{Observations}

Since 2011 , observations of solar radio emission at the UTR-2 radio telescope are carried out in the interferometric mode, which gives an opportunity for estimation of sizes and locations of solar radio burst sources. The UTR-2 radio telescope has an effective collecting area of up to $150000 \mathrm{~m}^{2}$ and it consists of 12 separate sections, which can be configured in different ways. For the discussed observations, the sections were grouped into three separate antennas: North (N), West (W) and South (S) antenna with 4 sections in each, thus forming a local interferometer with two orthogonal baselines B1 and B2 each being $674 \mathrm{~m}$ long (see Figure 1). These two baselines are inclined by $45^{\circ}$ to the meridian.

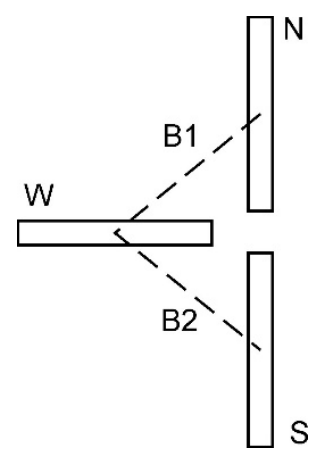

Fig. 1 The UTR-2 radio telescope as a two-base interferometer.

Two spectropolarimeters DSPZ (Zakharenko, et al. 2016) are used as a backend. These digital analyzers calculate complex correlation functions in an instantaneous frequency band of $10-32 \mathrm{MHz}$ for each of two bases. The absolute value of the correlation function carries information about the size of the source via a visibility function, while the phase of the correlation function is determined by the source angular displacement.

On 31 May 2013, a complex and powerful type II burst was observed by UTR-2, URAN-2 and NDA radio telescopes. The overall view of the burst is shown in Figure 2. The whole event started at 11:12 UT with the group of type III bursts. In this particular case, these type III bursts are the precursor of the following type II bursts and coincide in time with a solar flare of class C1.1 above the active region NOAA11761 (S18E23). The type II burst itself consists of two parts separated in time. First part lasted from 11:22:30 UT till 11:25:30 UT and the second one - from 11:28 UT till 11:39 UT. Both parts of the burst exhibit band splitting. The lanes were separated in frequency by $4.5 \mathrm{MHz}$ and $6 \mathrm{MHz}$, respectively. The dynamic spectrum obtained by the NDA shows also the harmonic structure of the bursts (see Figure 3). The burst appeared to be bounded both above at $65 \mathrm{MHz}$ and below at $10 \mathrm{MHz}$. The burst appeared to be extremely intense with maximum fluxes as high as 10000 s.f.u. at the frequency around $20 \mathrm{MHz}$ ( 1 solar flux unit (s.f.u.) equals $10^{-22} \mathrm{Wm}^{2} \mathrm{~Hz}^{-1}$ ).

No sign of a type II burst at lower frequencies was found by SWAVES receivers onboard the STEREO spacecraft.

This type II burst was apparently initiated by the CME recorded by the coronagraphs SOHO/LASCO-C2 at 12:12 UT (https://cdaw.gsfc.nasa.gov/CME_list/) and by STEREO-B/COR1 at 11:20 UT. The CME was not visible to the STEREO-A spacecraft.

\section{Analysis and Discussion}

\section{a) Determination of the direction and the speed} of the CME propagation using optical data

The views of the CME from the different locations of the satellites are shown in Figure 4. The CME of interest is marked with white arrows. The speeds of the CME measured from the SOHO and STEREO-B spacecraft are $280 \mathrm{~km} \cdot \mathrm{s}^{-1}$ and $340 \mathrm{~km} \cdot \mathrm{s}^{-1}$, respectively. Taking into account the longitudinal positions of STEREO-A $\left(138^{\circ}\right)$ and STEREO-B $\left(-141^{\circ}\right)$ spacecraft and the fact that this CME isn't visible to the former, we may conclude that this CME propagated at an angle of $\sim 30^{\circ}$ eastward to the line of sight from Earth with the velocity of about $340 \mathrm{~km} \cdot \mathrm{s}^{-1}$. In this case, the CME moves practically in the sky plane for the STEREO-B spacecraft and at $\sim 30^{\circ}$ eastward to the line of sight from the $\mathrm{SOHO}$ spacecraft. For such a trajectory, STEREO-A couldn't observe the CME because it was completely behind the Sun.

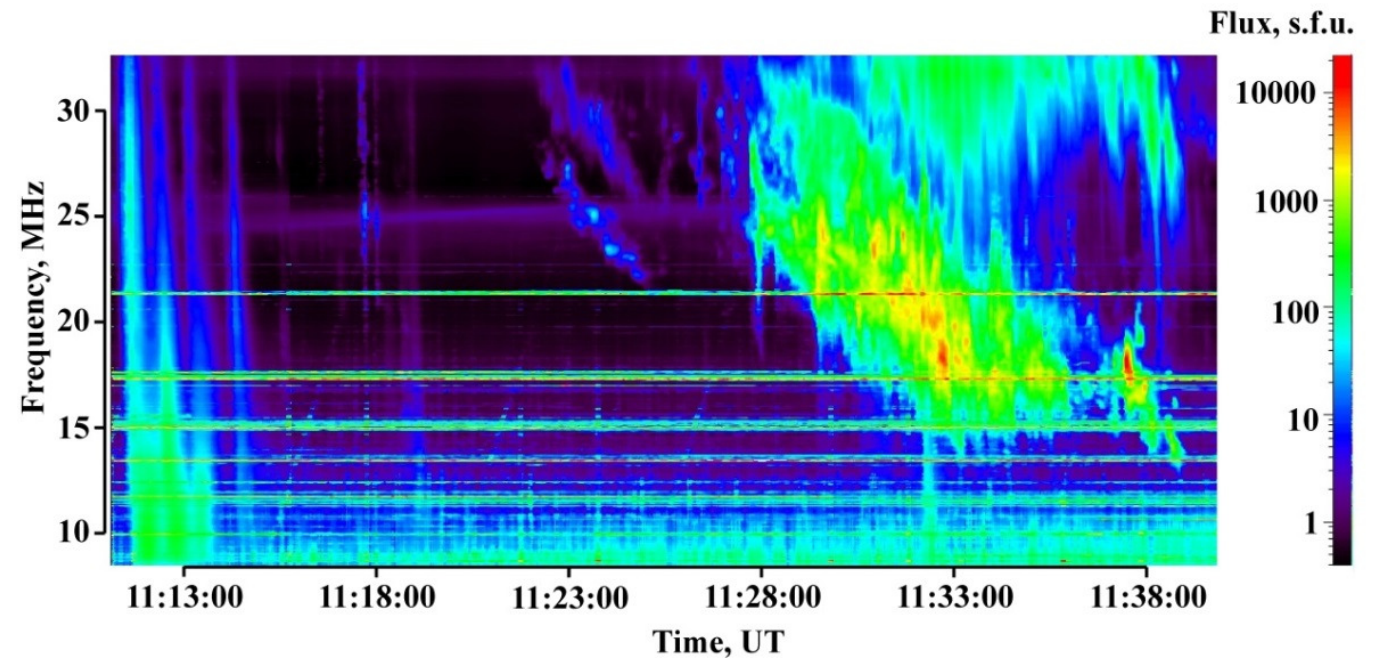

Fig. 2 Type II burst recorded on 31 May 2013 by the UTR-2 radio telescope. 


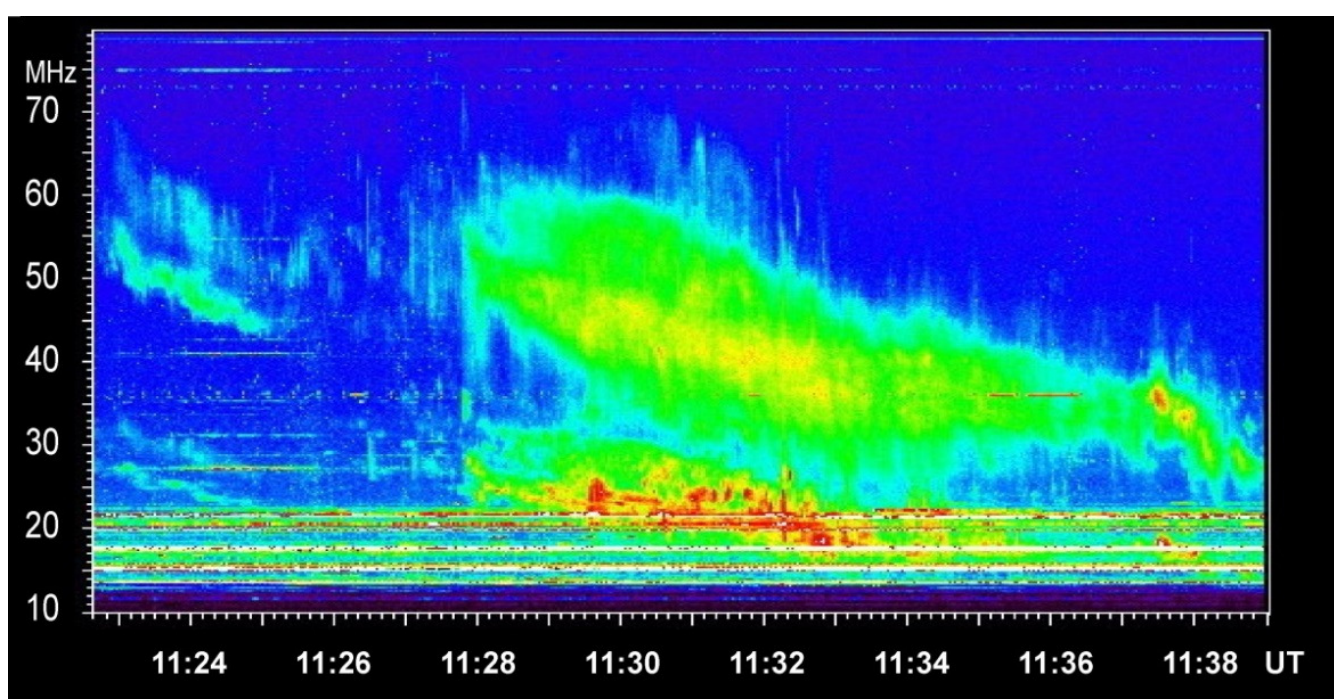

Fig. 3 Type II burst recorded on 31 May 2013 by the NDA radio telescope.



Fig. 4 Running difference images from STEREO-A (a), SOHO (b) and STEREO-B (c) spacecraft and the positions of the STEREO spacecraft on 31 May 2013 (d). The arrow in panels b and c points to the associated CME and in panel d marks the CME propagation direction.

b) Determination of the CME speed via the frequency drift rate of the type II bursts.

It is well known that because of the plasma emission mechanism, the frequency drift rate of any burst depends on the corona density profile and the source velocity.

For the current analysis, we apply the Newkirk (1961) corona density model. For the fundamental component of the discussed burst, the drift rate is not constant over the burst lifetime. In the beginning, the drift rate was about $-40 \mathrm{kHz} \cdot \mathrm{s}^{-1}$ corresponding to the radial velocity of about $800 \mathrm{~km} \cdot \mathrm{s}^{-1}$. The tail of the type II bursts had practically no drift. The radial velocity of the first part of the bursts is more than double to that of the CME speed according to coronagraph data. The possible reason of such a discrepancy is discussed below.

\section{c) Using spatial properties of the type II burst for retrieving the CME parameters}

The possibility of estimation of the radio sources sizes and positions using the UTR-2 radio telescope as an interferometer was shown in Shepelev (2015) and Melnik et al. (2017).

The size of the radio source in this case is determined through the visibility function (Thompson, et al. 1986)

$$
\gamma=\exp \left[-\left(\frac{\pi \theta_{L}}{2 \lambda \sqrt{2 n z}}\right)^{2}\right]
$$

where $\theta$ is the half-maximum angular size of the source, $L$ is the base length of the interferometer and $\lambda$ is the wavelength of the radio emission. Note that
Equation (1) is only valid for sources with a Gaussian brightness distribution. In the case when the interferometer is composed of the elements of the same array (providing identical antenna parameters) the visibility function can be calculated as (Shepelev, 2015):

$$
y=\frac{P_{9}}{\sqrt{P_{1} \cdot P_{2}}},
$$

where $\mathrm{P}_{\mathrm{c}}$ is the absolute value of the correlation function, and $P_{1}$ and $P_{2}$ are the power received by the two antennas of the interferometer base. Using equations (1) and (2) it is possible to get the source size along the direction of the baseline.

The phase of the correlation function allows to define the angular deflection of the source from the interferometer axis. We must note that the phase analysis is quite sensitive to the accuracy of the antenna beam pointing as well as to the refraction effects in the ionosphere. Thus the estimation of the source position with respect to the solar disc center is much less accurate than the estimation of the sources displacements with respect to each other. For the current analysis we assume the accuracy of the respective angular displacement measurement as about 1'. The absolute angular position of the source with respect to the center of the Sun is not worse than $8^{\prime}$ (one axis tick in Figures 5-8). Using the thermal radio emission of the quiet Sun as the reference point can substantially increase the latter accuracy. Since the angular size of the quiet radio Sun at frequencies around $20 \mathrm{MHz}$ is roughly $1^{\circ}$, additional shorter baselines should be used. Such experiments are planned in future. 
The spatial properties of the first part of the type II burst, which manifests a well-distinguished band splitting, are shown in Figure 5.

We have analyzed the spatial properties of the two lanes of the first type II burst taken at the same moment of time. The source of the higher frequency lane is drawn in red, while lower frequency lane is marked with a blue color. The source sizes are almost equal (14' and $16^{\prime}$ respectively) and this gives us the effective brightness temperatures of these sources of $2 \cdot 10^{12} \mathrm{~K}$ and $4 \cdot 10^{11} \mathrm{~K}$. The obtained sizes of the type II bursts sources appeared to be slightly smaller than the sizes of type III bursts, measured by the same radio telescope with the similar method at the same frequencies (Melnik, et al. 2017). Former analyses of the spatial properties of the solar radio sources by the UTR2 radio telescope were concentrated exclusively at the source size estimation (Shepelev, 2015, Melnik, et al. 2017). These analyses are based on the amplitude of the cross-correlation function, which is less affected by the accuracy of the radio telescope pointing and the refraction effects. Current experiment is the first attempt to estimate the displacement of the source center using the phase of cross-correlation function at the UTR-2 radio telescope.

From the analysis of the respective angular positions of the radio sources it is evident that the higher frequency lane is situated closer to the Sun than the lower frequency lane. In addition, we must note that these two lanes have a different morphology as can be easily seen in Figure 5b. It is evident that the higher frequency lane consists of "clouds" extended along the frequency axis (vertically), while the lower frequency lane is composed of exclusively horizontally stretched structures. These facts show that the sources of the two lanes are different and thus speak in favor of the upstream and downstream model of band splitting of type lls.

Since the three methods of the CME displacement estimations were mentioned in the beginning of this section it seems interesting to roughly compare the results provided by each of them. Let's take the type II burst segment at 11:25 UT (Figure 5) for such a comparison. At this time the heliocentric height of the nose of the CME according to the STEREOBEHIND/COR 1 image was $1.87 R_{\odot}$, where $R_{\odot}$ is the solar radius. The frequencies of the upper and lower lanes of the type II burst correspond to the heights of $1.76 R_{\odot}$ and $1.88 R_{\odot}$, respectively, assuming the Newkirk corona density model. And finally interferometric observations give the projections of these heights to the sky plane equal to $1.6 R_{\odot}$ and $1.7 R_{\odot}$. It is evident that obtained values are of the same order of magnitude taking into account the declared accuracy. The angular sizes of the sources appeared to be much larger than their angular displacements thus making accurate determination of linear velocity difficult. Using baselines of different lengths might improve the situation in further experiments.

In the same way we have analyzed how the source of the lower-frequency lane moves in space with time. Apparently the source moves away from the Sun (Figure 6). The velocity obtained from this displacement is around $900 \mathrm{~km} \cdot \mathrm{s}^{-1}$.

This velocity is very close to that obtained from the frequency drift rate and much higher than the velocity provided by the coronagraphs. To our opinion this discrepancy may possibly be connected with migration of the radio source across the shock surface. Indeed, the obtained size of the radio source is much less than the size of the CME itself and thus the source may change its position at the shock with time causing the apparent increase of its velocity.

In such an assumption the source may migrate from the flank of the CME toward its nose at the beginning of the type II burst, where the velocities retrieved from the drift rate and from the interferometer are higher than that provided by the coronagraph. On the contrary, the source possibly migrates backwards at the tail of the type II burst where the burst has practically no drift. Another possible reason of the discrepancy could be extremely irregular shape of the source that leads to errors in the detection of the source center position. Interferometer at the UTR-2 estimates the sizes and the locations only along the two baselines of equal lengths and thus is sensitive to the shape of the source. Current configuration of the UTR-2 radio telescope provides accurate estimations of the spatial parameters of the spherical sources with Gaussian brightness distribution.


b)

Fig. 5 The positions and sizes of the sources of type II burst with band splitting (a) and the corresponding dynamic spectrum (b). 

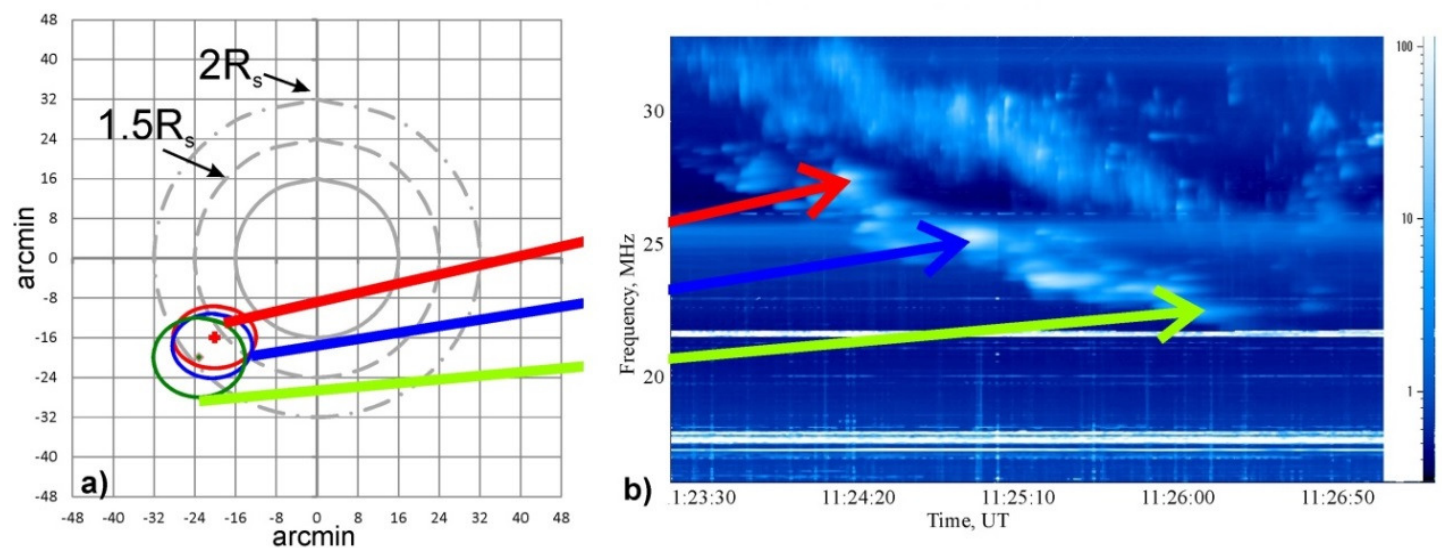

Fig. 6 Displacement of the type II burst source with time (a) and the corresponding dynamic spectrum (b).


Fig. 7 Displacement of the reverse HB sub-bursts source with time (a) and the corresponding dynamic spectrum (b).
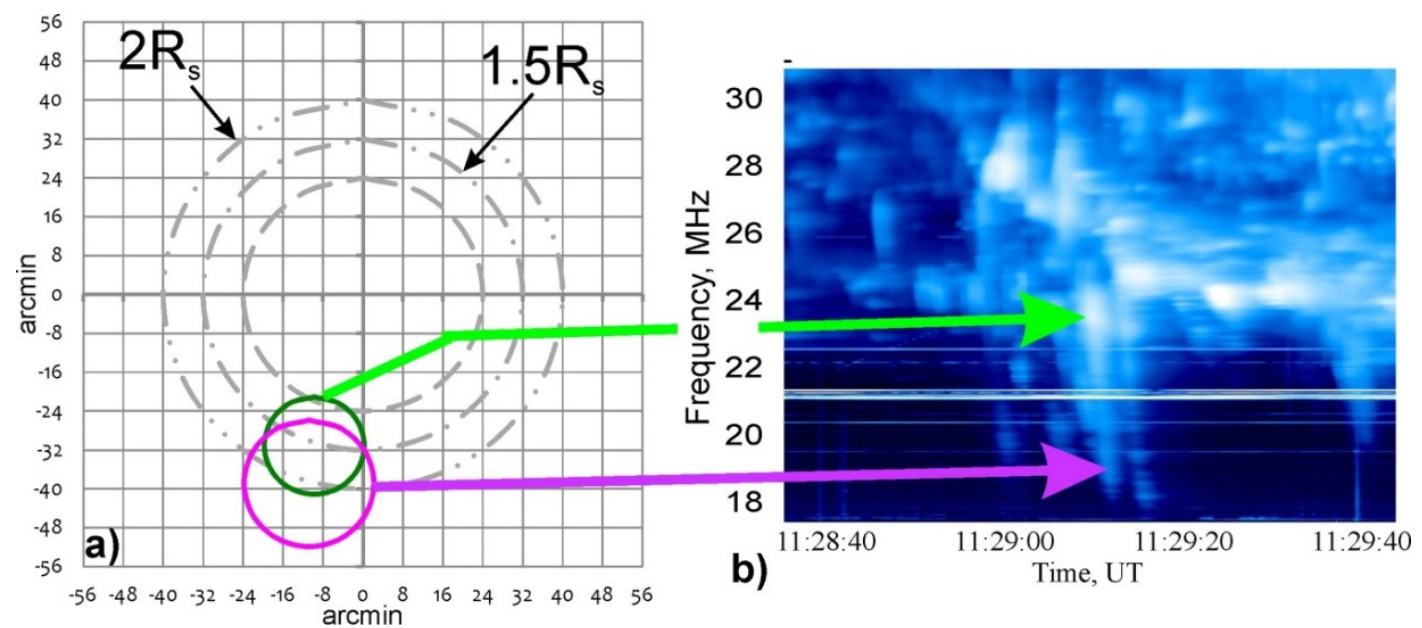

Fig. 8 Displacement of the forward HB sub-bursts source with time and the corresponding dynamic spectrum (b).

The formation of the HB structure is generally attributed to the electron beams acceleration at the shock in two opposite directions [Cairns and Robinson, 1987]. Figures 7 and 8 evidently illustrate the inward and outward movement of the sources of HB subbursts with opposite frequency drift signs. The velocities of these movements are about $0.3 c$, and are close to the type III electrons velocity.

\section{d) Polarization properties of the type II burst.}

Data from the URAN-2 radio telescope allow to measure the polarization of radio emission. For this particular burst, the fundamental HB sub-bursts reveal a high degree of circular polarization reaching as high as $70 \%$. The harmonic counterparts are weakly polarized. This is in good agreement with theoretical view, according to which the fundamental radio emission is the o-mode wave with almost $100 \%$ circular polarization, while the harmonic emission is a mixture of 
the 0 - and $x$-mode waves resulting in much less polarization degree (Melrose, 1987). On the contrary, both the fundamental and the harmonic components of the first part of the type II burst (without a HB structure) are completely unpolarized. This may indicate that the sources of different parts of type II burst are located at different sites with different conditions.

\section{Conclusions}

Present work has shown the possibility of detecting of spatial properties of fine structured solar radio bursts, such as type II bursts with band splitting and a HB structure. The speeds of the sources of different parts of the complex type II burst were obtained by two independent methods: by the frequency drift rate (model dependent value) and by the source spatial displacement (model independent value). The obtained velocities, $800 \mathrm{~km} / \mathrm{s}$ and $900 \mathrm{~km} / \mathrm{s}$ respectively, appeared to be very close. At the same time they both differed considerably from the associated CME speed, obtained from coronagraphic data. This effect could be possibly connected with migration of the radio burst source across the surface of the shock.

The sizes of the type II burst appeared to be much smaller than the apparent sizes of the CME with which they are associated. They are also slightly smaller than normal type III bursts sources. High flux densities and small sizes result in extremely high brightness temperatures of the type II burst source, which in few cases reach the value of about $10^{14} \mathrm{~K}$.

Obtained locations of the burst sources indicate that band splitting is likely the result of emission from regions ahead and behind the shock front. They also show that the HB structure is most probably the result of accelerated electrons escaping the shock towards and away from the Sun.

The obtained spatial properties of the type II burst sources also point out that different fragments of the type II burst of the same frequency may originate from different parts of the shock surface. This fact may lead to uncertainty in the source speed measurements using frequency drift rates of type II bursts.

\section{Acknowledgements}

The work was partially performed under the support of the European FP-7 project SOLSPANET (F9P7-People2010-IRSES-269299).

\section{References}

Cairns, I.H. and Robinson, R.D.: 1987, Solar Phys. 111, 365. DOI 10.1007/BF00148526.

Dorovskyy, V.V., Melnik, V.N., Konovalenko, A.A., Brazhenko, A.I., Panchenko, M., Poedts, S., and Mykhaylov, V.A.: 2015, Solar Phys. 290, 2031. DOI: 10.1007/s11207-015-0725-9.

Gopalswamy, N., Aguilar-Rodriguez, E., Yashiro, S., Nunes, S., Kaiser, M.L., and

Howard, R.A.: 2005, J. Geophys. Res.: Space Phys. 110, A12S07. DOI: $10.1029 / 2005 J A 011158$.

Melnik, V.N., Konovalenko, A.A., Rucker, H.O., Stanislavsky, A.A., Abranin, E.P., Lecacheux, A., Mann, G., Warmuth, A., Zaitsev, V.V., $\quad$ Boudjada, M.Y., $\quad$ Dorovskii, V.V., Zaharenko, V.V., Lisachenko, V.N., and Rosolen, C.: 2004, Solar Phys., 222, 151. DOI: 10.1023/B:SOLA.0000036854.66380.a4.

Melnik, V.N., Shepelev, V.A., Brazhenko, A.I., Dorovskyy, V.V., Rucker, H.O., and Poedts, S.: 2017, Sun and Geosphere, 12, 105.

Melrose, D.B.: 1987, Solar Phys., 111, 89. DOI: 10.1007/BF00145443

Nelson, G.J. and Melrose, D.B.: 1985, Solar radiophysics. Studies of emission from the Sun at meter wavelengths, Cambridge University Press, Cambridge-New-York, p. 333.

Newkirk, G.J.: 1961, Astrophys. J., 133, $983 . \quad$ DOI: $10.1086 / 147104$.

Shepelev, V.A.: 2015, Radiophys. \& Radioastron. (in Russian), 20, 20.

Thompson, A.R., Moran, J.M., and Swenson, G.W.: 1986, Interferometry and Synthesis in Radio Astronomy, WileyInterscience, New York, USA.

Vršnak, B., Magdalenić, J., and Zlobec, P.: 2004, Astron. \& Astrophys., 413, 753. DOI: 10.1051/0004-6361:20034060.

Zakharenko, V., Konovalenko, A., Zarka, P., Ulyanov, O., Sidorchuk, M., Stepkin, S., Koliadin, V., Kalinichenko, N., Stanislavsky, A., Dorovskyy, V., and 42 coauthors.: 2016, J. Astron. Instrumentation, 5, 1641010-738. DOI: $10.1142 / \mathrm{S} 2251171716410105$. 